\title{
Calcificaciones cerebrales típicas como orientación de cuadro clínico inespecífico
}

\author{
S. BENITO CONEJERO, C. DÍAZ ESPEJO, J. M. LÓPEZ DOMÍNGUEZ, E. PUJOL \\ DE LA LLAVE \\ Servicio de Medicina Interna y Sección de Neurología. Hospital Juan Ramón Jiménez. \\ Huelva
}

\section{RESUMEN}

La enfermedad celíaca es una enteropatía por sensibilidad al gluten de origen autoinmune, considerada clásicamente como enfermedad exclusivamente gastrointestinal. Posteriormente se han puesto de manifiesto manifestaciones extraintestinales. Un número importante de síndromes neurológicos catalogados de idiopáticos al inicio, han sido descritos en el contexto de enfermedad celiaca, presentando respuesta total o parcial tras dieta sin gluten. Se ha descrito asociado a enfermedad celiaca un tipo muy característico de calcificaciones cerebrales occipitales, que a veces forma parte de un síndrome específico llamado síndrome de Gobby. Presentamos una paciente con enfermedad celiaca oligosintomática, que presentaba las peculiares calcificaciones cerebrales que fueron halladas tras realización de una prueba de imagen al presentar migraña rebelde a tratamiento, y que orientaron hacia su diagnóstico. La migraña mejoró sustancialmente tras realizar dieta sin gluten, como en otros casos descritos en la literatura. El hecho de que existan manifestaciones neurológicas asociadas a enfermedad celiaca hace posible la reversibilidad de las mismas tras dieta exenta de gluten.

PALABRAS CLAVE: Enfermedad celiaca. Gluten. Sensibilidad al gluten. Calcificaciones cerebrales.
CEREBRAL CALCIFICATIONS: A CLUE FOR A DIAGNOSTIC PROCESS IN A INESPECIFIC CLINICAL CASE.

\begin{abstract}
Coeliac disease is a gluten sensitive enteropathy, autoimmune in origin, which has been traditionally regarded as a gastrointestinal disease. Years later it has been reported an extraintestinal affection. A huge number of neurological syndromes of unknown cause had been initially described in association with coeliac disease, with total or parcial response to a gluten free-diet. A specific kind of occipital cerebral calcifications in relation to coeliac disease has been also described, and sometimes it means the existence of a syndrom called "Gobby's Syndrom".

We show a patient with a mild unknown coeliac disease, a woman who had occipital cerebral calcifications in a TAC cerebral, which was made because of her wild migraine and that it leaded the diagnosis. The migraine disappeared after a gluten free-diet, like similar cases reported by literature. The fact of existing neurological symtoms associated to coeliac diseases opens a therapeutc window of opportunity because they would repond to a gluten free-diet.
\end{abstract}

KEY WORDS: Coeliac disease. Gluten. Gluten sensitive. Cerebral calcifications.

Benito Conejero S, Díaz Espejo C, López Domínguez JM, Pujol de la Llave E. Calcificaciones cerebrales típicas como orientación de cuadro clínico inespecífico. An Med Interna (Madrid) 2006; 127-129.

\section{INTRODUCCIÓN}

La enfermedad celiaca es una enteropatía por sensibilidad al gluten de origen autoinmune, que cursa típicamente con malabsorción de nutrientes secundario a lesión inflamatoria de mucosa de intestino delgado, y que se caracteriza por mejoría y resolución clínica e histológica tras retirada de gluten de la dieta. El diagnóstico es clínico y serológico y se confirma biopsia. El espectro clínico de la enfermedad coeliaca es amplio y por ello esta entidad se encuentra infradiagnosticada, sobre todo en pacientes cuyo debut no es el gastrointestinal habitual, incluso pudiendo debutar con alguna manifestación extraintestinal (1). Entre las manifestaciones neurológicas más frecuentesdescritas en enfermedad celiaca están ataxia y neuropatía periférica, depresión, epilepsia y migraña (2-7). El hallazgo más característico en las técnicas de neuroimagen son las calcificaciones cerebrales (8-11). Describimos una paciente que cumplía criterios de migraña con aura visual en la que, la detección de dichas calcificaciones permitió establecer el diagnóstico de enfermedad celiaca e instaurar una dieta sin gluten, que resolvió las manifestaciones clínicas.

\section{CASO APORTADO}

Mujer de 40 años que presentaba cefalea de 20 años de evolución con características de migraña con aura visual, con una frecuencia de 13 episodios mensuales. La paciente carecía de antecedentes patológicos 
de interés, salvo ocasionales episodios de diarrea. autolimitada desde su infancia. La exploración general y neurológica fueron normales. Se le practicó analítica general incluyendo: hemograma, VSG, coagulación, bioquímica completa con hormonas tiroideas, calcio, fósforo y PTH, que fueron normales. El ECG y Rx de tórax y abdomen se encontraban dentro de la normalidad. La TAC craneal con y sin contraste que mostró calcificaciones occipitalesbilaterales de tipo giriforme (Figs. 1 y 2). La RNM de cráneo con y sin gadolinio confirmó los mismos hallazgos de la TAC. El EEG fue normal.

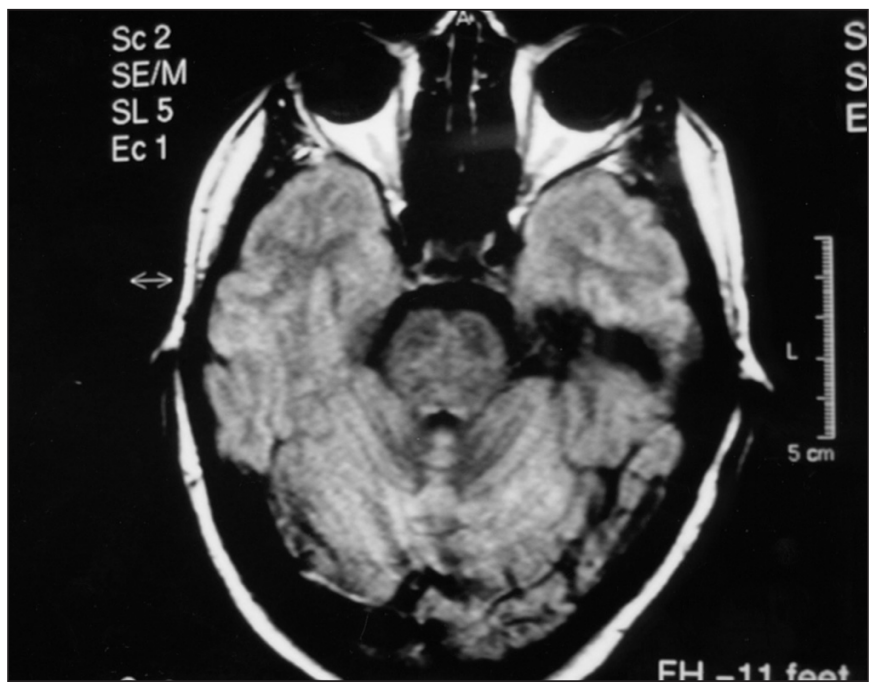

Fig. 1. TAC de cráneo que muestra las típicas calcificaciones occipitales bilaterales de tipo giriformes en una paciente con enfermedad celíaca.

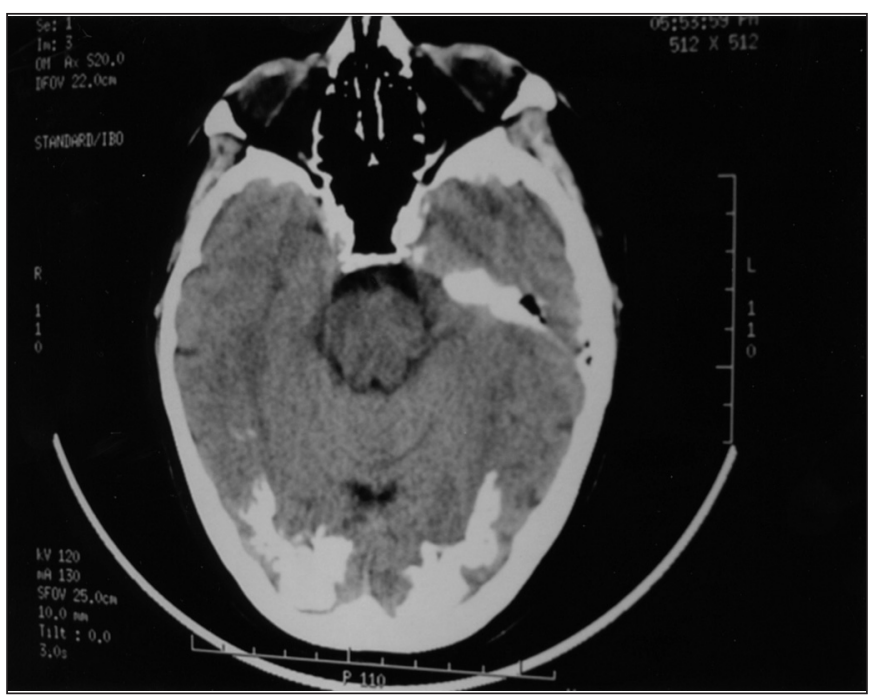

Fig. 2. RNM de cráneo de la misma paciente.

Dado que la paciente presentaba clínica de diarrea desde la infancia, se practicó determinación de anticuerpos antigliadina y antiendomisio que resultaron positivos. Entonces se llevó a cabo biopsia duodenal que mostró atrofia de mucosa intestinal con hiperplasia de criptas y aumento del número de linfocitos presentes intraepiteliales, estableciéndose el diagnóstico de enfermedad celiaca.

Se prescribió una dieta exenta de gluten. Tras varios meses de seguimiento los accesos diarreicos habían desaparecido, la paciente había ganado peso y no había presentado nuevas crisis de migraña.

\section{DISCUSIÓN}

Se estima que hasta un $10 \%$ de los pacientes con enfermedad celiaca desarrollan manifestaciones neurológicas $(2,3)$. Entre ellas se han descrito: ataxia, neuropatía periférica, miopatía, ataxia conmioclonus, mielopatía, demencia, migraña, depresión y otras manifestaciones psiquiátricas,epilepsia, síndrome del túnel del carpo, infarto isquémico cerebral, meningitis, síndromesmielocerebrales desmielinizantes y degeneración cerebelosa (2-7). Las más frecuentes manifestaciones neurológicas en enfermedad celiaca son ataxia y neuropatía periférica en una serie (2) y depresión, epilepsia y migraña en otra (3). Un número importante de síndromes neurológicos catalogados deidiopáticos al inicio, han sido descritos en el contexto de sensibilidad al gluten, por lo que sesugiere en la literatura la conveniencia de realizar screening serológico de enfermedad celiaca en los pacientes con manifestaciones neurológicas de origen desconocido (8).

En el caso que presentamos la paciente se encontraba oligosintomática desde el punto de vista digestivo. El diagnóstico se sospechó al observar en la TAC craneal practicada por su migraña las calcificaciones occipitales bilaterales de tipo giriforme características (9-11). Es sabida la estrecha relación de la enfermedad celiaca con las calcificaciones cerebrales, aunque la frecuencia exacta no se ha determinado. Se especula que aproximadamente la mitad de los pacientes con enfermedad celiaca presentan calcificaciones cerebrales (12). Existe de igual modo una alta prevalencia de enfermedad celiaca en pacientes con calcificaciones cerebrales (11). La mayoría de pacientes conenfermedad celiaca con calcificaciones cerebrales presentan además epilepsia (2,9-12). En 1988Sammaritano es el primero en proponer la existencia de un síndrome específico, que incluye calcificaciones intracraneales, enfermedad celiaca y crisis parciales, tras aparición de casos anecdóticos que asocian dichas condiciones (10). Posteriormente Gobby publica la más amplia serie de pacientes con esta triada que tomó su nombre como Síndrome de Gobby (9).

La prevalencia de migraña en una cohorte de 388 casos con enfermedad coeliaca fue $3,1 \%$ (5). Lo que podría parecer una asociación casual no lo es, pues en la mayoría de los casos la migraña desaparece al instaurar una dieta sin gluten $(4,5)$, al igual que sucedió en nuestra paciente; y ello sugiere una relación causal entre la enfermedad celiaca y la migraña.

Se han postulado diferentes teorías sobre la patogenia de las manifestaciones neurológicas en los pacientes con enfermedad celiaca. Un mecanismo sería el déficit de nutricional vitamínico y de oligoelementos secundario, y otro sería autoinmune mediante reacción cruzada de los anticuerpos contra el gluten con los epítopos de las células de purkinje. En exámenes patológicos se ha hallado infiltrado inflamatorio perivascular y pérdida parcheada de células de purkinje (2$4,7,10)$. El predominio en determinada población (italiana), sugiere una predisposición genética (12).

Nuestro propósito es señalar la asociación de calcificaciones occipitales bilaterales y enfermedad celiaca. Además, la posibilidad de que la enfermedad celiaca se asocie a manifestacionesneurológicas como la migraña, que en principio son 
consideradas idiopáticas, ampliándose con ello el espectro de presentación de la enfermedad celiaca clásica, como se hace hincapié en la literatura.

Esto supone en ocasiones que se trate de manifestacio- nes potencialmente reversibles tras dieta sin gluten. Consideramos que la determinación de anticuerpos antigliadina y antiendomisio son un buen test de screening en situaciones clínicas de sospecha enfermedad celiaca previo a biopsia.

\section{Bibliografía}

1. Mäki M, Collin P.Coeliac disease. Lancet 1997; 349: 1755-59.

2. Hadjivassiliou M, Grunewald RA, Davies-Jones GA.Gluten sensibility as a neurological illness. J Neurol Neurosurg Psychiatry 2002; 72: 560-563.

3. Pegiran Tengah DSNA, Wills AJ, Colmes GKT. Neurological complications of coeliac disease. Postgrad Med J 2002; 78: 393-398.

4. Wills AJ, Unsworth DJ.The neurology of gluten sensibility: separating the wheat from the chaff. Curr Opin Neurol 2002; 15: 519-523.

5. Hadjivassiliou M, Grunewald RA, Lawden M. et al. Headache and CNS white matter abnormalities associated with gluten sensitivity. Neurology 2001; 56: 385-388.

6. Finelli PF, McEntee WJ, Ambler M, et al. Adult coeliac disease presenting as cerebellar syndrome. Neurology 1980; 30: 245-9.

7. Holmes GKT. Neurological and psychiatric complications in coeliac disease. In: Gobby G, Anderman F, Naccarato S, et al,eds. Epilepsy and other neurological disorders in coeliac disease. London: John Libbey, 1997.

8. Hadjivassiliou M, Gibson A, Davies-Jones GA, et al. Does cryptic gluten sensitivity play a part in neurological illness? Lancet 1996; 347: 369-71.

9. Gobby G, Bouquet F, Greco L, et al. Coeliac disease, epilepsy, and cerebral calcifications. Lancet 1992; 340: 439-43.

10. Sammaritano M, Andermnn F, Melanson D, et al. The syndrome of intractable epilelepsy, bilateral occipital calcifications, and folic acid deficiency. Neurology 1988; 38 (Supl 1): 239.

11. Magaudda A, Dalla Bernardina, P De Marco, et al. Bilateral occipital calcification, epilepsy and coeliac disease: clinical and neuroimaging features of a new syndrome. J Neurol Neurosurgy Pisychiatry 1993; 56: 885-889.

12. Díaz RM, González-Rabelino G, Delfino A. Epilepsia, calcificaciones cerebrales y enfermedad celíaca. Importancia del diagnóstico precoz. Revista De Neurología 2005; 40: 417-420. 